\title{
Septicemia mortality reduction in neonates in a heart rate characteristics monitoring trial
}

\author{
Karen D. Fairchild', Robert L. Schelonka², David A. Kaufman', Waldemar A. Carlo ${ }^{3}$, John Kattwinkel', Peter J. Porcelli', \\ Cristina T. Navarrete ${ }^{5}$, Eduardo Bancalari ${ }^{5}$, Judy L. Aschner ${ }^{6}$, M. Whit Walker ${ }^{7}$, Jose A. Perez ${ }^{8}$, Charles Palmer ${ }^{9}$, Douglas E. Lake ${ }^{10}$, \\ T. Michael O'Shea ${ }^{4}$ and J. Randall Moorman ${ }^{10}$
}

BACKGROUND: Abnormal heart rate characteristics (HRC) wax and wane in early stages of culture-positive, late-onset septicemia (LOS) in patients in the neonatal intensive care unit (NICU). Continuously monitoring an HRC index leads to a reduction in mortality among very low birth weight (VLBW) infants. We hypothesized that the reduction in mortality was due to a decrease in septicemia-associated mortality.

METHODS: This is a secondary analysis of clinical and HRC data from 2,989 VLBW infants enrolled in a randomized clinical trial of HRC monitoring in nine NICUs from 2004 to 2010.

RESULTS: LOS was diagnosed 974 times in 700 patients, and the incidence and distribution of organisms were similar in HRC display and nondisplay groups. Mortality within $30 \mathrm{~d}$ of LOS was lower in the HRC display as compared with the nondisplay group (11.8 vs. $19.6 \%$; relative risk: 0.61 ; $95 \%$ confidence interval: $0.43,0.87 ; P<0.01)$, but mortality reduction was not statistically significant for patients without LOS. There were fewer large, abrupt increases in the HRC index in the days leading up to LOS diagnosis in infants whose HRC index was displayed.

CONCLUSION: Continuous HRC monitoring is associated with a lower septicemia-associated mortality in VLBW infants, possibly due to diagnosis earlier in the course of illness.

ate-onset septicemia (LOS, presenting beyond $3 \mathrm{~d}$ of age) Lis a leading cause of death among very low birth weight (VLBW; i.e., infants weighing $<1,500 \mathrm{~g}$ ) infants $(1,2)$. Survivors of LOS have longer durations of mechanical ventilation, longer hospital stays, and worse neurodevelopmental outcomes as compared with gestational age-matched infants who do not develop sepsis $(3,4)$. Efforts toward reducing the toll of neonatal sepsis include preventive strategies, biomarker measurements for earlier detection, and use of adjunct immunotherapies. Extensive investigation into sepsis biomarkers has yielded promising candidates such as inflammatory cytokines (5), acute-phase proteins, and leukocyte activation markers (6), but the drawback of these blood tests is that they are available on a limited basis and are usually obtained when a patient is already sick, even if only subtly so. Immune-modulating therapies given after a patient develops signs of sepsis have also not proven beneficial $(7,8)$. Treatment with antibiotics or adjunct therapies before overt clinical deterioration might lead to better outcomes. The challenge is to identify patients early in transition from being well to being sick; continuous monitoring of heart rate characteristics (HRC) was developed for this purpose $(9,10)$.

Alterations in HRC occur as part of the pathophysiologic response to systemic infection. The autonomic nervous system modulates heart rate, with frequent small accelerations and decelerations occurring in response to sympathetic and parasympathetic input to cardiac pacemaker cells. Decreased beat-to-beat variability and transient decelerations occur in acute pathologic conditions such as fetal asphyxia and neonatal sepsis $(11,12)$. In patients in the neonatal intensive care unit (NICU), we have shown that changes in HRC wax and wane before the onset of clinical signs of sepsis $(9,12,13)$, raising the possibility of earlier sepsis detection and treatment through display of HRC monitor data to clinicians. Earlier institution of antibiotics and supportive therapies could curb the systemic inflammatory response linked to tissue damage and mortality in sepsis.

Continuous HRC monitoring translates reduced heart rate variability and transient decelerations to estimate the fold increase in probability of imminent sepsis (HRC index) using mathematical algorithms (14-17). The impact of HRC monitoring on outcomes was tested in a randomized clinical trial of 3,003 VLBW infants in nine NICUs (18). The trial had a pragmatic design in that clinicians were given information about how the HRC index was developed and were instructed that an elevation in the HRC index should prompt a bedside evaluation and consideration of appropriate blood tests or treatment, but no specific course of action was mandated in response to

\footnotetext{
'Department of Pediatrics, University of Virginia, Charlottesville, Virginia; ${ }^{2}$ Department of Pediatrics, University of Oregon Health Science Center, Portland, Oregon;

${ }^{3}$ Department of Pediatrics, University of Alabama at Birmingham, Birmingham, Alabama; ${ }^{4}$ Department of Pediatrics, Wake Forest University, Winston-Salem, North Carolina;

${ }^{5}$ Department of Pediatrics, University of Miami/Jackson Memorial Hospital, Miami, Florida; ${ }^{6}$ Department of Pediatrics, Vanderbilt University, Nashville, Tennessee; ${ }^{7}$ Department of Pediatrics, Greenville Hospital System, Greenville, South Carolina; ${ }^{8}$ Department of Pediatrics, Winnie Palmer Children's Hospital, Orlando, Florida; ${ }^{9}$ Department of Pediatrics, Pennsylvania State University, University Park, Pennsylvania; ${ }^{10}$ Department of Medicine, University of Virginia, Charlottesville, Virginia. Correspondence: Karen D. Fairchild (Kdf2n@virginia.edu)
} 
the HRC index (18). Importantly, there was a $22 \%$ relative reduction in mortality among infants whose HRC index was displayed to clinicians $(10.2-8.1 \% ; P=0.04)$. Here, we focused on infants in the clinical trial who had one or more episodes of LOS and tested the hypothesis that mortality within $30 \mathrm{~d}$ of LOS is lower in the HRC display as compared with the nondisplay group. To explore the possibility that HRC monitoring led to earlier diagnosis of LOS, we analyzed large, abrupt increases, or spikes, in the HRC index and also tested the hypothesis that LOS was diagnosed after fewer spikes when the HRC index was displayed.

\section{RESULTS}

\section{Septicemia Incidence and Clinical Characteristics}

Of 3,003 VLBW infants randomized, 2,989 fulfilled the inclusion criteria and were included in the original analysis $(1,500$ HRC display and 1,489 nondisplay). Fourteen patients were excluded from analysis due to withdrawal of consent, birth weight $\geq 1,500 \mathrm{~g}$, or cardiac arrhythmias (18). Overall, 9,882 blood cultures were obtained (5,225 from 1,020 infants in the HRC display group and 4,657 from 980 infants in the nondisplay group), and there were 974 episodes of LOS in 700 patients (Figure 1). The majority of LOS episodes (70\%) were caused by Gram-positive bacteria, with coagulase-negative Staphylococcus (CONS) accounting for 49\%. The 974 episodes of LOS were equally distributed among patients in the HRC display and nondisplay groups (486 in 348 infants and 488 in 352 infants, respectively). There were no significant differences in the distribution of organism classes causing septicemia in the two groups (Figure 1). As compared with infants without LOS, those who experienced one or more episodes were of lower birth weight and gestational age (Table 1). In the HRC display and nondisplay groups, there were no significant differences in birth weight, gestational age, or gender for infants with septicemia as compared with those without. Infants with one or more episodes of LOS in the HRC display group had

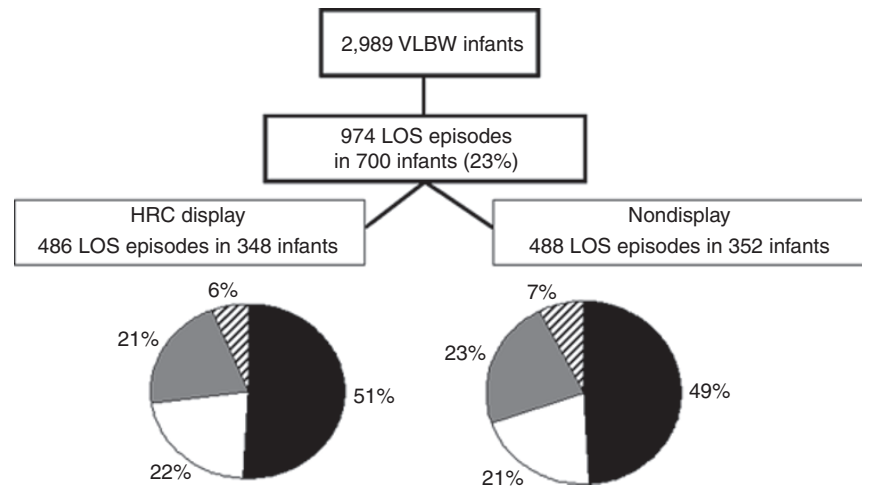

Figure 1. Late-onset septicemia (LOS) in a randomized clinical trial of heart rate characteristics (HRC) monitoring in very low birth weight (VLBW) infants. Overall, 2,989 VLBW infants at 9 neonatal intensive care units had HRC monitoring and were randomized to having their HRC index displayed to clinicians or not displayed. Twenty-three percent of infants had LOS ( $>3 \mathrm{~d}$ of age), and the organism distribution is shown for infants randomized to HRC display (left) or nondisplay (right). Black, coagulasenegative Staphylococcus; white, other Gram-positive bacteria; gray, Gramnegative bacteria; and stripes, fungus. more days alive and not on a ventilator (the primary end point of the clinical trial) as compared with those in the nondisplay group (76.4 vs. $69.7 \mathrm{~d}$; $P=0.048$ ). Infants with LOS in the HRC display group also had more antibiotic days as compared with those in the nondisplay group (32.1 vs. $29.0 \mathrm{~d} ; P=0.047$ ).

\section{Septicemia-Related Mortality}

Mortality within $30 \mathrm{~d}$ of LOS was significantly lower in HRC display as compared with nondisplay infants (11.8 vs. $19.6 \%$; relative risk: $0.61 ; 95 \%$ confidence interval: $0.43,0.87 ; P<$ 0.01 ), as was overall in-hospital mortality (15.2 vs. $21.9 \%$; relative risk: $0.70 ; 95 \%$ confidence interval: $0.51,0.96 ; P<$ 0.03). Mortality within $7 \mathrm{~d}$ of LOS was $9.2 \%$ in HRC display as compared with $13.9 \%$ in nondisplay infants (relative risk: 0.66; 95\% confidence interval: $0.43,1.01 ; P=0.059$ ). Within each organism group, there was reduced mortality with HRC display (Figure 2). The mortality reduction reached statistical significance for CONS, the most frequent infecting organism (6.3 vs. $18.1 \%$ for HRC display vs. nondisplay; $P=0.0003$ ). For infants who did not have septicemia, the mortality reduction from $6.6 \%$ in the nondisplay group to $6.0 \%$ in the HRC display group was not statistically significant (Table 1 ). In the randomized trial, there were 30 excess deaths in the nondisplay as compared with the HRC display group, and 27 of these excess deaths occurred within $30 \mathrm{~d}$ of LOS.

\section{Heart Rate Characteristics Index in Episodes of LOS}

The HRC index increased in the days leading up to clinical diagnosis of LOS, with a significantly higher HRC index at $24 \mathrm{~h}$ as compared with $48 \mathrm{~h}$ before LOS diagnosis $(P<0.01)$ (Figure 3a). By contrast, the concurrent baseline probability of sepsis, calculated from the established risk factors of gestational and postmenstrual age, birth weight, and presence of mechanical ventilation showed only a small increase due to some patients requiring intubation and mechanical ventilation around the time of septicemia. There was an increase in the average HRC index before the clinical diagnosis of LOS with CONS, other Gram-positive bacteria, Gram-negative bacteria, and fungi. Peak HRC index was similar for each pathogen group (CONS: $3.0 \pm 2.2$; other Gram-positive bacteria: 3.1 \pm 2.3 ; Gram-negative bacteria: $3.0 \pm 2.2$; fungus: $3.3 \pm 2.2$; values are given in mean $\pm \mathrm{SD} ; P=0.68$ ). Ninety two percent of cases of LOS in nondisplay infants were associated with $\mathrm{HRC}$ index $>1$ and $79 \%$ with $\mathrm{HRC}$ index $>2$ within $24 \mathrm{~h}$ of diagnosis.

Infants in the nondisplay group had a significantly higher average HRC index in the 2-7 d period leading up to the time of positive blood culture as compared with infants in the HRC display group $(P<0.01$ for each day from -2 to -7 relative to LOS) (Figure 3b). Figure 4 illustrates the occurrence of a single abrupt rise in the HRC index ("spike") around the time of LOS vs. multiple spikes in the days leading up to diagnosis. The proportion of patients with spikes at nonseptic times was comparable between the two groups (Table 2). By contrast, more patients in both groups had spikes in the 2-7-d time interval before the diagnosis of LOS, demonstrating that the spikes are 


\section{Articles | Fairchild et al.}

Table 1. Clinical characteristics and outcomes of VLBW infants with and without septicemia in HRC display and nondisplay groups

\begin{tabular}{|c|c|c|c|c|c|c|}
\hline & \multicolumn{3}{|c|}{ Septicemia } & \multicolumn{3}{|c|}{ No septicemia } \\
\hline & HRC display $(n=348)$ & Nondisplay $(n=352)$ & $P$ & HRC display $(n=1,152)$ & Nondisplay $(n=1,137)$ & $P$ \\
\hline Birth weight (g) & $813(237)$ & $787(238)$ & 0.149 & $1,055(271)$ & $1,047(276)$ & 0.52 \\
\hline Male (\%) & 49 & 49 & 0.628 & 49 & 50 & 0.62 \\
\hline Hospital stay (days) & $92(55)$ & $86(48)$ & 0.130 & $56(37)$ & $56(40)$ & 0.98 \\
\hline Days alive not on MV & $76.4(43)$ & $69.7(46)$ & 0.048 & $107(31)$ & $106(33)$ & 0.67 \\
\hline In-hospital mortality \% ( $n)$ & $15.2(53)$ & $21.9(77)$ & 0.026 & $6.0(69)$ & $6.6(75)$ & 0.61 \\
\hline 30-d LOS mortality \% ${ }^{\mathrm{a}}(n)$ & $11.8(41)$ & $19.6(68)$ & 0.005 & & & \\
\hline
\end{tabular}

Mean (standard deviation) or \%, where indicated. Bold $P$ values are statistically significant at $P<0.05$.

HRC, heart rate characteristics; LOS, late-onset septicemia; MV, mechanical ventilation; VLBW, very low birth weight.

a30 days from last septicemia episode.

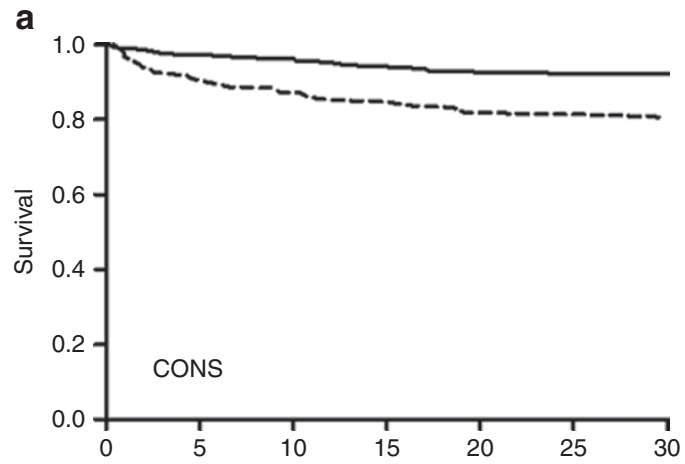

b
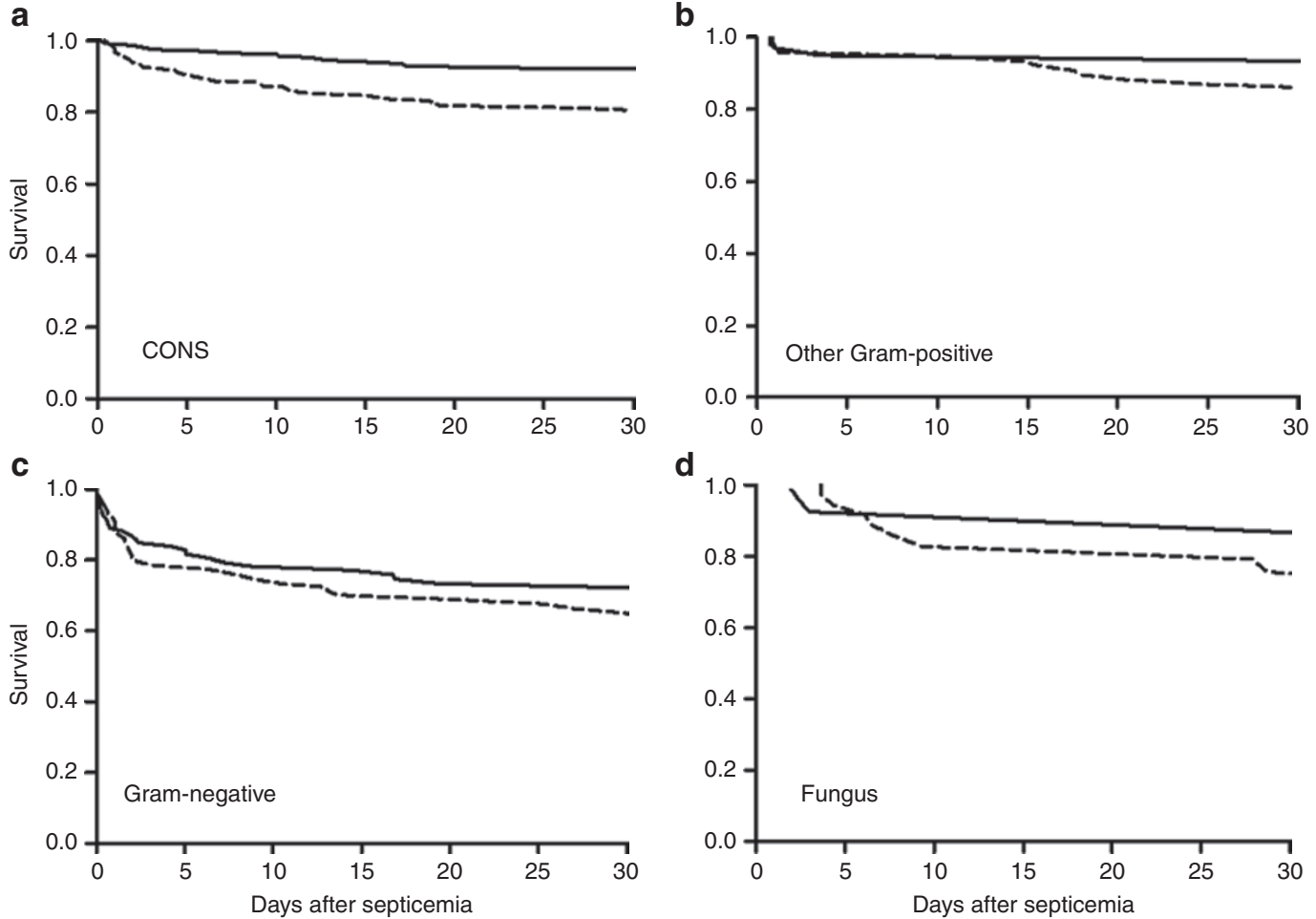

Figure 2. Organism-specific mortality based on heart rate characteristics (HRC) monitor display. Survival is shown within $30 \mathrm{~d}$ of diagnosis of late-onset septicemia with (a) CONS, (b) other Gram-positive bacteria, (c) Gram-negative bacteria, (d) and fungi. Survival was higher in each organism group in infants with HRC displayed (solid line) as compared with those with HRC not displayed (dashed line). CONS, coagulase-negative Staphylococcus.

relevant to septicemia. Furthermore, more patients in the nondisplay group had large spikes prior to LOS diagnosis than those in the HRC display group regardless of the infecting organism.

\section{DISCUSSION}

In this secondary analysis of data from a large, randomized clinical trial of HRC monitoring in VLBW infants, we found that mortality reduction was much greater for infants with septicemia when the HRC index was displayed to clinicians than for infants without septicemia. In addition, we found that the average HRC index increased before the clinical diagnosis of LOS, irrespective of infecting organism.
Sepsis-related morbidity and mortality are unacceptably high in part because the diagnosis is often delayed by the subtlety of presenting signs. New biomarker tests for sepsis improve on earlier ones (6) but require clinical suspicion of illness to prompt blood testing. By then, the stage of illness may be so advanced that antibiotic administration fails to curtail the systemic inflammatory response that can lead to death and disability. Adjunct sepsis therapies such as intravenous immunoglobulin given to neonates once they show clinical signs of infection have not proven effective in reducing mortality and morbidity (7). Continuous monitoring of HRC has the potential advantage of alerting clinicians to pathophysiologic 
a

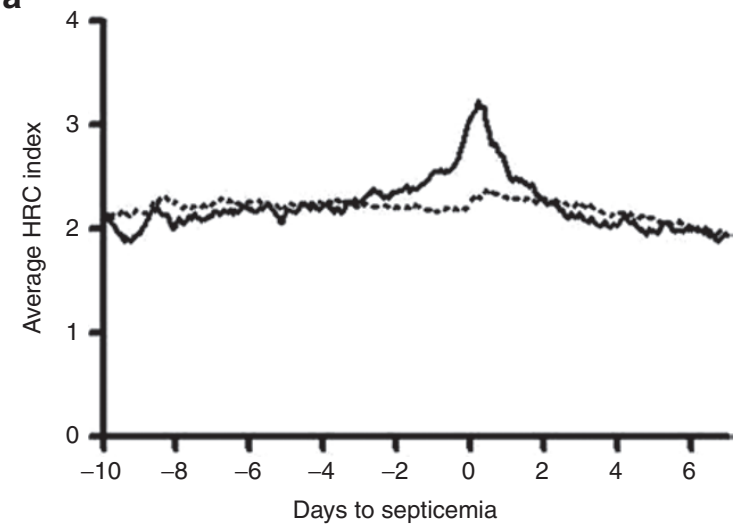

b

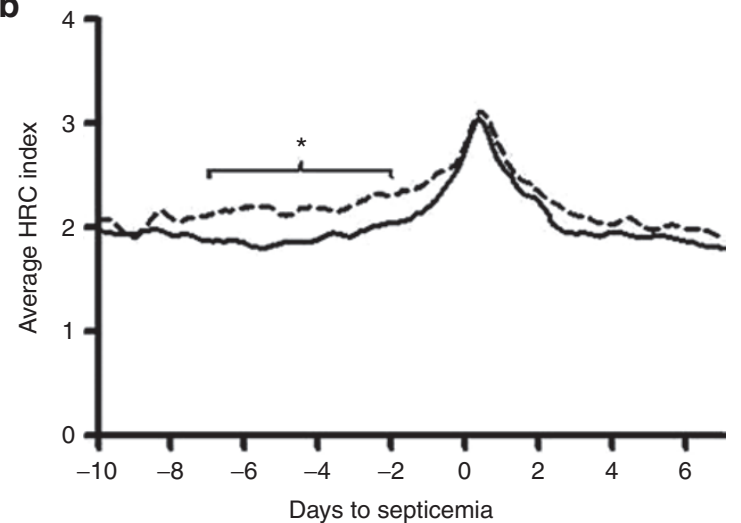

Figure 3. Heart rate characteristics index versus risk factor-based probability and HRC display versus nondisplay in cases of late-onset septicemia. (a) Average HRC index (solid line) in the 2-wk period around the diagnosis of LOS at day 0 , as compared with the risk-factor based probability of sepsis (dotted line) based on gestational and postmenstrual age, birth weight, and mechanical ventilation. (b) Average HRC index around the diagnosis of LOS in infants randomized to HRC display (solid line) or nondisplay (dashed line) $\left({ }^{*} P<0.01\right)$.

changes in the early phase of sepsis, before overt clinical deterioration, leading to earlier treatment and improved outcomes.

In this large trial of VLBW infants, the incidence of sepsis was comparable with that reported in other large surveys (1) and was very similar in HRC display and nondisplay groups, underscoring that the function of HRC monitoring is not sepsis prevention, but rather early detection. The HRC index increased in the day before the clinical diagnosis of LOS with Gram-positive or Gram-negative bacteria or fungi. The average HRC index was significantly higher in the week leading up to LOS diagnosis in infants whose HRC index was not displayed to clinicians and was accompanied by more acute HRC index spikes. We have previously described waxing and waning of abnormal HRC for several days before the clinical presentation of sepsis in NICU patients $(9,12,13)$. There is no way to determine exactly when an infant develops a bloodstream infection, so we can only speculate that these prediagnosis HRC spikes represent an early phase of illness and that recognition, evaluation, and treatment at this time averted clinical deterioration and contributed to lower morbidity and mortality. Among the 700 infants who developed sepsis in the randomized trial, those a

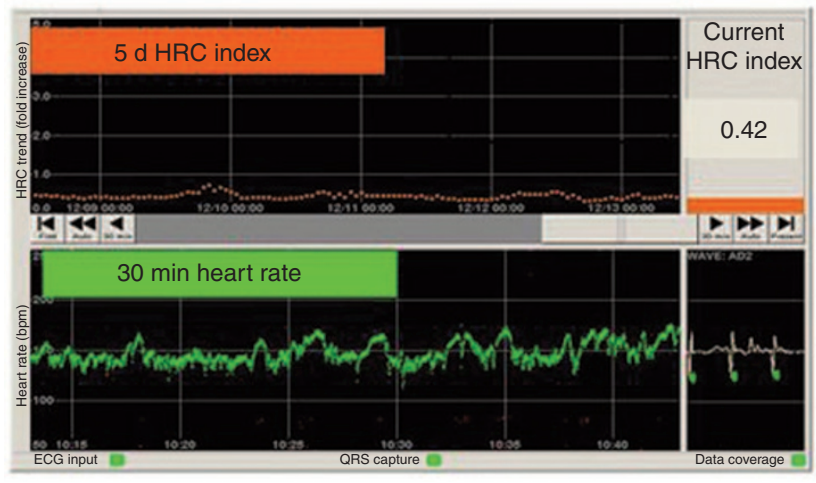

b

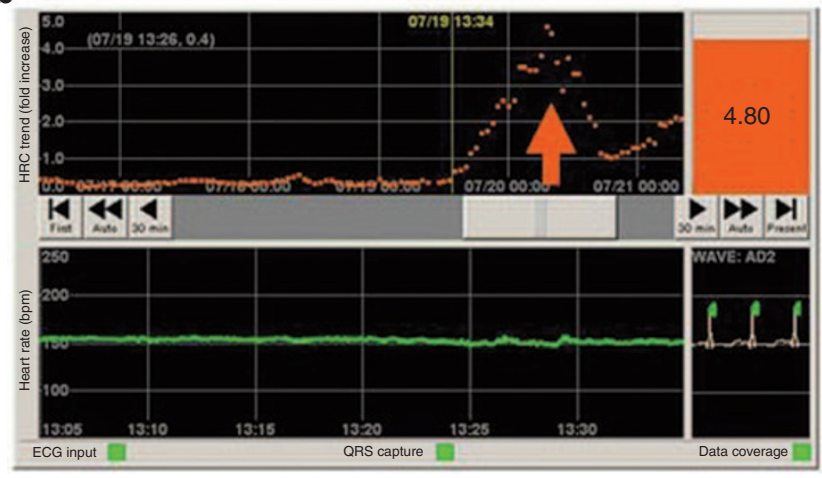

C

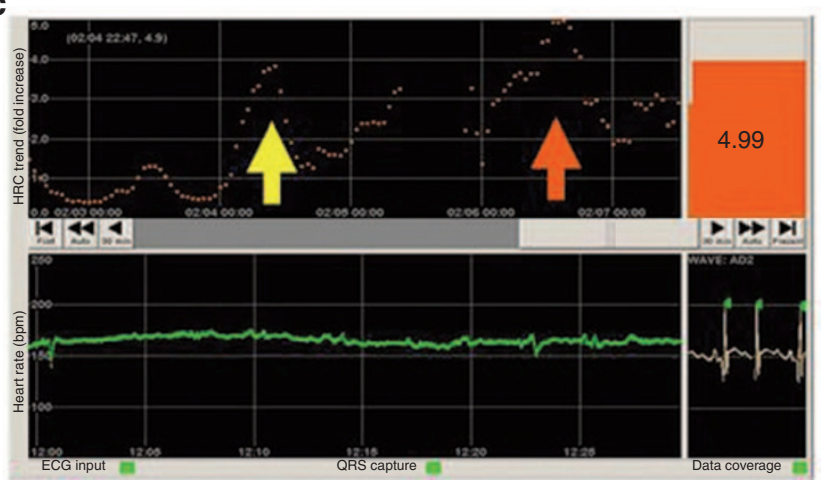

Figure 4. Heart rate characteristics (HRC) index monitor screen shots illustrating normal HRC and large HRC index spikes. (a) HRC monitor individual patient display shows the 5-d HRC index trend (orange), the latest $30 \mathrm{~min}$ of heart rate in beats per minute (bpm) (green), and the current HRC index (0.42). In this infant, the HRC index has been low $(<1)$ for at least $5 \mathrm{~d}$, indicating normal heart rate characteristics and low probability of sepsis in the next $24 \mathrm{~h}$. (b) HRC monitor display for an infant with a large HRC index spike at the time of septicemia diagnosis (orange arrow, HeRO score: 4.80) and no large spikes in the days before. (c) Large HRC index spike at the time of septicemia diagnosis (orange arrow, HeRO score: 4.99) and a large HRC index spike $2 \mathrm{~d}$ before sepsis diagnosis (yellow arrow, HeRO score: 3.89). HeRO, heart rate observation.

whose HRC index was displayed to clinicians had not only fewer acute increases in HRC index and lower mortality but also significantly more days alive and not on a ventilator, which was the primary end point of the original study. Lower mortality among HRC display infants occurred across all organism classes, with the largest reduction occurring with CONS septicemia, which accounted for nearly half of all LOS cases. The trade-off for lower mortality is that infants whose HRC index 
Table 2. Percentage of patients with large HRC index spikes 2-7 days prior to diagnosis of late-onset septicemia (LOS), or during random 5-day periods

\begin{tabular}{lccc}
\hline & $\begin{array}{c}\text { HRC display } \\
\text { \% with spike }\end{array}$ & $\begin{array}{c}\text { Nondisplay } \\
\text { \% with spike }\end{array}$ & \multicolumn{1}{c}{$P$} \\
\hline Never septic & 8.6 & 7.0 & 0.47 \\
Remote from LOS & 16.1 & 17.6 & 0.66 \\
LOS, all organisms & 22.4 & 34.3 & $<\mathbf{0 . 0 1}$ \\
CONS & 22.4 & 36.8 & $<\mathbf{0 . 0 1}$ \\
Other Gram-positive bacteria & 21.1 & 31.0 & 0.17 \\
Gram-negative bacteria & 18.2 & 26.5 & 0.22 \\
Fungus & 33.3 & 46.7 & 0.79
\end{tabular}

Bold $P$ values are statistically significant at $P<0.05$.

CONS, coagulase-negative Staphylococcus; HRC, heart rate characteristics.

aMore than a week before or after LOS.

was displayed had 12\% more blood cultures obtained and 11\% more days on antibiotics. The fact that there were, on average, only three extra antibiotic days in the infants in the HRC display as compared with the nondisplay group among infants who developed LOS leads us to speculate that monitoring the HRC index may give clinicians insight into appropriate timing of both initiating and discontinuing antibiotics.

The HRC monitoring study is the largest randomized clinical trial in VLBW infants reported to date. A limitation of the current analysis of LOS in this trial is that the exact timing of onset of sepsis relative to the changes in the HRC index is unknown. The design of the clinical trial did not include a mandated intervention for infants with an acute increase in HRC index, and we can only speculate as to whether the increases in the HRC index in the days leading up to the positive blood culture represent a subclinical sepsis prodrome and an opportunity for earlier intervention.

In conclusion, HRC monitoring in VLBW infants reduces mortality, and this reduction in mortality is associated with a lower mortality following late-onset sepsis. It is possible that HRC monitoring prompts recognition of sepsis in its earlier stages when treatment is more likely to reduce morbidity and mortality.

\section{METHODS}

\section{Patient Population}

VLBW infants at nine NICUs were enrolled in a randomized clinical trial of HRC monitoring from April 2004 to May 2010. The study was sponsored by the National Institute of Child Health and Human Development and Medical Predictive Science Corporation (Charlottesville, VA). Institutional review boards approved the protocol at each study site, and informed consent was obtained from parents for infants' participation. In this trial, all infants had their HRC index monitored and were randomized 1:1 (stratified by birth weight $\geq$ or $<1,000 \mathrm{~g}$ ) to having their HRC index continuously displayed or not displayed to nurses, nurse practitioners, and physicians.

\section{Clinical Data Collection, and Sepsis and Mortality Definitions}

In the randomized clinical trial, we recorded demographics, dates and results of blood cultures, days on antibiotics or antifungal agents (other than prophylactic fluconazole or ampicillin), days on mechanical ventilation, and date of death or NICU discharge. The current analysis results from a careful reclassification of septicemia episodes in the trial with LOS was defined as: (i) a positive blood culture obtained at $>3 \mathrm{~d}$ of age, excluding corynebacterium and diphtheroids, (ii) no prior positive blood culture for the same organism in the previous 7 $\mathrm{d}$, and (iii) antibiotics administered for at least $5 \mathrm{~d}$ when the organism grown was CONS. "Other positive" blood cultures were reclassified if a specific organism could be identified through database review. The 18 cases of multiple organisms in a blood culture were classified by what was considered the most virulent organism. We excluded 15 episodes from the organism-specific analyses because the organism could not be identified through database review. We also excluded 92 episodes in which there was a positive blood culture for CONS but the infant received fewer than $5 \mathrm{~d}$ of antibiotics. As a result of this analysis, 37 patients were reclassified as having no sepsis.

Mortality was assessed as all in-hospital mortality and as death within $30 \mathrm{~d}$ and within $7 \mathrm{~d}$ of the last episode of LOS.

\section{Analysis of Risk Factor-Based LOS Probability}

The HRC index was originally conceived, developed, and validated for the assessment of probability of imminent LOS in VLBW infants $(12,19-21)$. To establish the baseline probability of LOS based on standard risk factors (1), we developed a model incorporating gestational and postmenstrual age, birth weight, and presence of mechanical ventilation in the patients in the control, nondisplay group.

\section{HRC Index Monitoring and Spike Analysis}

The US Food and Drug Administration 510(k)-cleared Heart Rate Observation monitoring system was provided by Medical Predictive Science Corporation (Charlottesville, VA). The monitor uses existing electrocardiogram or heart rate data from standard bedside monitors to calculate the HRC index, which is the fold increase in probability that the patient will have a clinical deterioration consistent with sepsis in the next $24 \mathrm{~h}$. The HRC index is derived from an externally validated logistic regression expression that relates decreased heart rate variability and transient heart rate decelerations to the probability that the baby will imminently be diagnosed with sepsis. The monitor displays the current HRC index, which is updated every hour and represents HRC over the previous $12 \mathrm{~h}$. The monitor also displays the 5-d HRC index trend.

We analyzed the average hourly HRC index around the time of LOS in infants in the HRC display and nondisplay groups. To examine a possible mechanism of lower mortality with HRC index monitoring, we also counted large, abrupt increases in the HRC index ("spikes") in the 2-7-d period before the clinical diagnosis of LOS (the time period during which there was a significant difference in average HRC index in HRC display vs. nondisplay infants). We defined a spike as an increase in HRC index of at least 3 compared with the prior 5-d running average, excluding the $12 \mathrm{~h}$ before the peak. Rates of large spikes were compared in infants who never developed LOS and in infants in the HRC display and nondisplay groups who developed LOS, both during a randomly chosen nonseptic $5-\mathrm{d}$ period and in the $2-7-\mathrm{d}$ period before LOS diagnosis.

\section{Statistical Analysis}

HRC index is reported as mean \pm SD. We used multivariable logistic regression modeling adjusted for repeated measures using the HuberWhite method and determined confidence intervals by bootstrap (22). Differences in demographics, sepsis rates, and organism types were tested for significance using nonparametric tests. Differences in survival after sepsis diagnosis were tested for significance using a proportional hazards model. Statistical testing was performed with Matlab software (MathWorks, Natick, MA), with a two-tailed level of significance of 0.05 .

\section{STATEMENT OF FINANCIAL SUPPORT}

J.R.M. was supported by National Institutes of Health (Bethesda, MD) grant R01-HD48562.

Disclosure: J.R.M. and D.E.L. have consulting agreements and equity shares in Medical Predictive Science Corporation, Charlottesville, VA. The other authors declare no conflict of interest. 


\section{REFERENCES}

1. Stoll BJ, Hansen N, Fanaroff AA, et al. Late-onset sepsis in very low birth weight neonates: the experience of the NICHD Neonatal Research Network. Pediatrics 2002;110(2 Pt 1):285-91.

2. Makhoul IR, Sujov P, Smolkin T, Lusky A, Reichman B; Israel Neonatal Network. Pathogen-specific early mortality in very low birth weight infants with late-onset sepsis: a national survey. Clin Infect Dis 2005;40:218-24.

3. Schlapbach LJ, Aebischer M, Adams M, et al.; Swiss Neonatal Network and Follow-Up Group. Impact of sepsis on neurodevelopmental outcome in a Swiss National Cohort of extremely premature infants. Pediatrics 2011;128:e348-57.

4. Stoll BJ, Hansen NI, Adams-Chapman I, et al.; National Institute of Child Health and Human Development Neonatal Research Network. Neurodevelopmental and growth impairment among extremely low-birth-weight infants with neonatal infection. JAMA 2004;292:2357-65.

5. Raynor LL, Saucerman JJ, Akinola MO, Lake DE, Moorman JR, Fairchild KD. Cytokine screening identifies NICU patients with Gram-negative bacteremia. Pediatr Res 2012;71:261-6.

6. Ng PC, Ang IL, Chiu RW, et al. Host-response biomarkers for diagnosis of late-onset septicemia and necrotizing enterocolitis in preterm infants. J Clin Invest 2010;120:2989-3000.

7. Brocklehurst P, Farrell B, King A, et al. Treatment of neonatal sepsis with intravenous immune globulin. N Engl J Med 2011;365:1201-11.

8. Carr R, Modi N, Dore C. G-CSF and GM-CSF for treating or preventing neonatal infections. Coch Database Syst Rev 2003;2003:CD003066.

9. Fairchild KD, O'Shea TM. Heart rate characteristics: physiomarkers for detection of late-onset neonatal sepsis. Clin Perinatol 2010;37:581-98.

10. Fairchild $\mathrm{K}$, Aschner JL. HeRO monitoring to reduce mortality in NICU patients. Res Reports Neonatol 2012;2:65-76.

11. Griffin MP, Lake DE, Bissonette EA, Harrell FE Jr, O'Shea TM, Moorman JR. Heart rate characteristics: novel physiomarkers to predict neonatal infection and death. Pediatrics 2005;116:1070-4.
12. Griffin MP, O'Shea TM, Bissonette EA, Harrell FE Jr, Lake DE, Moorman JR. Abnormal heart rate characteristics preceding neonatal sepsis and sepsis-like illness. Pediatr Res 2003;53:920-6.

13. Moorman JR, Lake DE, Griffin MP. Heart rate characteristics monitoring for neonatal sepsis. IEEE Trans Biomed Eng 2006;53:126-32.

14. Griffin MP, Scollan DF, Moorman JR. The dynamic range of neonatal heart rate variability. J Cardiovasc Electrophysiol 1994;5:112-24.

15. Cao H, Lake DE, Griffin MP, Moorman JR. Increased nonstationarity of neonatal heart rate before the clinical diagnosis of sepsis. Ann Biomed Eng 2004;32:233-44

16. Kovatchev BP, Farhy LS, Cao H, Griffin MP, Lake DE, Moorman JR. Sample asymmetry analysis of heart rate characteristics with application to neonatal sepsis and systemic inflammatory response syndrome. Pediatr Res 2003;54:892-8.

17. Lake DE, Richman JS, Griffin MP, Moorman JR. Sample entropy analysis of neonatal heart rate variability. Am J Physiol Regul Integr Comp Physiol 2002;283:R789-97.

18. Moorman JR, Carlo WA, Kattwinkel J, et al. Mortality reduction by heart rate characteristic monitoring in very low birth weight neonates: a randomized trial. J Pediatr 2011;159:900-6.e1.

19. Griffin MP, Moorman JR. Toward the early diagnosis of neonatal sepsis and sepsis-like illness using novel heart rate analysis. Pediatrics 2001;107: 97-104.

20. Griffin MP, Lake DE, Moorman JR. Heart rate characteristics and laboratory tests in neonatal sepsis. Pediatrics 2005;115:937-41.

21. Griffin MP, Lake DE, O'Shea TM, Moorman JR. Heart rate characteristics and clinical signs in neonatal sepsis. Pediatr Res 2007;61: 222-7.

22. Harrell FE. Regression Modeling Strategies: With Applications to Linear Models, Logistic Regression and Survival Analysis. Berlin: Springer, 2001. 